\title{
Grippeimpfung und Autonomie
}

\author{
U. Thurnherr
}

1 Eine Alternative zur Impfung wird unter spezifischen Umständen durch eine Prävention mit antiviralen Medikamenten geboten.

2 Vgl. Müller DA. Nationale Grippepräventionskampagne Weitere Erfolge zeichnen sich ab. In diesem Heft.

3 Panchaud C. Grippeimpfung. Ein Abwägen von Interessen. Krankenpflege 2003;10:24.

Korrespondenz:

Prof. Dr. phil. Urs Thurnherr

Pädagogische Hochschule

Postfach 111062

D-76060 Karlsruhe

E-Mail:

urs.thurnherr@ph-karlsruhe.de
Der wesentlichste Grund, warum sich das Personal im Medizinalbereich gegen Grippe impfen lassen sollte, besteht darin, dass es mit Risikopatientinnen und -patienten in Kontakt kommt oder kommen könnte, bei denen eine Ansteckung mit Grippe zu schwerwiegenden Komplikationen und unter Umständen gar zum Tode führen kann. Zum angesprochenen Personal im Medizinalbereich sind alle Berufsgruppen $\mathrm{zu}$ zählen, die im Spital, in der Arztpraxis, im Heim oder in der Hauspflege unmittelbar mit Patientinnen und Patienten $\mathrm{zu}$ tun haben. Dazu gehören sowohl die Ärzteschaft und das Pflegepersonal als auch andere Gruppen, wie beispielsweise die Physio- oder Ergotherapeutinnen und -therapeuten sowie das Reinigungspersonal. Wenn im Kontext der Grippe von Risikopatientinnen und -patienten die Rede ist, so sind dabei Menschen gemeint, die an Herz-Kreislauf- oder Atemwegserkrankungen, an Immundefizienz u.a. leiden. Solche Patientinnen und Patienten können gewiss nicht nur durch das Personal, sondern jederzeit auch durch andere Patientinnen und Patienten bzw. Mitbewohnerinnen und Mitbewohner oder auch durch Besucherinnen und Besucher mit Grippe angesteckt und entsprechend gefährdet werden. Mit Sicherheit wäre es von Vorteil, wenn alle Kontaktpersonen von Risikopatientinnen und -patienten und diese selber geimpft wären. Die Ansteckungsgefahr für diese Patientinnen und Patienten könnte aber nicht zuletzt gemindert werden, wenn vor allem auch das Personal sich impfen lassen würde [1].

\section{Krankheiten vorbeugen, andere schützen, vor Schaden bewahren}

Faktisch ist die Bereitschaft zur Grippeimpfung auf seiten des Personals im Gesundheitsbereich bis heute nicht besonders gross [2]. Von einem medizinethischen Standpunkt aus könnte es demgegenüber überlegenswert sein, ob die Grippeimpfung nicht eine moralische Verpflichtung darstellt. Denn mit Blick auf die in Frage kommenden Grundsätze - wie zum Beispiel seine Patientinnen und Patienten soweit wie möglich zu schützen bzw. sie vor Schaden zu bewahren - scheint die eigene Grippeimpfung moralisch geboten zu sein. Dieser Überlegung halten manche Mitglieder des Personals entgegen, dass die Impfung immerhin einen Eingriff in den eigenen Körper darstellt und es zur Freiheit gehöre, sich gegebenenfalls nicht impfen lassen zu wollen. In einem Artikel hat Catherine Panchaud das betreffende Problem aus Sicht des Pflegepersonals auf die Formel gebracht: «Freiheit gegen Schutz des anderen» [3]. Die Frage drängt sich aber auf, zu welcher Entscheidung ich kommen werde, wenn ich meine Freiheit in moralischem Sinne gebrauche und in dem Bereich meine Autonomie verwirkliche.

\section{Was heisst Autonomie?}

Autonomie in philosophisch-ethischer Bedeutung kann als moralische Selbstbestimmung bzw. Freiheit bezeichnet werden. Selbstbestimmung im Bereich des Moralischen besteht darin, dass ich gemäss meinen Moralvorstellungen selber bestimme, was ich tun soll. Auf eine moralische Frage gebe ich eine Antwort im Sinne meiner Moral. Impfverweigerung aus Desinteresse am Problem genügt von daher im vorliegenden Kontext von vornherein als Antwort nicht. Moralische Fragen beschäftigen sich damit, wie unsere Welt zu gestalten ist, damit sie zu einer humanen Welt wird. Wenn ich mithin meine Angelegenheiten autonom bestimme, versuche ich festzulegen, nach welchen begründbaren, mithin vernünftigen Normen wir meiner Ansicht nach leben sollen, damit wir unsere Lebenswelt human einzurichten vermögen. Meine Autonomie erfüllt sich schliesslich darin, dass ich mich zu den Gegebenheiten meiner Lebenssituation, zu meinen Wünschen und Hoffnungen sowie zu den anderen Menschen und zur Aussenwelt insgesamt in ein gestaltendes Verhältnis setze, wobei das Gestaltungsprinzip jeweils ein selber gegebenes «Gesetz» der Menschlichkeit bildet. Autonomie, d.i. Selbstgesetzgebung, kann von daher auch als vernünftige Freiheit bezeichnet werden. Vor diesem Hintergrund scheint die Autonomie von mir zu fordern, mich gegen Grippe impfen zu lassen, wenn ich dadurch das Risiko für andere Menschen vermindere, an einer Komplikation in der Folge von Grippe gar zu sterben: Mit meiner Entscheidung zur Impfung verwirkliche ich sodann ein Stück Menschlichkeit. 


\section{Gegenargumente}

Betrachtet man den argumentativen Aufwand gegen die Grippeimpfung selbst von seiten des Medizinalpersonals, kann die Antwort auf die Frage nach einer möglichen moralischen Verpflichtung zur Grippeimpfung scheinbar doch nicht ganz so einfach sein. Gerne möchte ich hier ein paar der öfter angeführten Argumente gegen die Impfung genauer prüfen, um zu sehen, ob die Sache der Autonomie nicht überzeugender durch diese Argumente vertreten wird. Dazu sollten wir sämtliche relevanten Informationen heranziehen, welche die Grippeimpfung betreffen. Denn Autonomie ist durchgehend mit einer gewissen Pflicht verbunden, sich zu informieren.

\section{Autonomie und Information}

Es dürfte vernünftig sein, seine Entscheidung auf alle zur Verfügung stehenden Informationen abzustützen und nicht einfach einer augenblicklichen Stimmung nachzugeben. Zur gemeinsamen Hochhaltung von Autonomie gehören deshalb die spezifischen Verpflichtungen, zum einen Informationen bereitzustellen und zum anderen Informationen einzuholen. Nur wer wirklich informiert ist, kann tatsächlich in sinnvoller Weise selber bestimmen. Demgegenüber kommt die Grippepräventionskampagne des Bundesamtes für Gesundheit (BAG) nicht zuletzt der korrespondierenden Pflicht nach, die Menschen in adäquater Form zu informieren. Die Erfüllung der Informationspflicht im Respekt vor der Autonomie desjenigen, der informiert werden soll, sieht sich mit ganz spezifischen normativen Forderungen konfrontiert. Um nur einige dieser informationsethischen Normen zu nennen: Der Informierende muss vorab ehrlich sein, und seine Informationen müssen sachlich dargeboten werden. Ferner muss der Informierende fachkundig sein und dabei möglichst umfassend informieren. Die gelieferten Informationen müssen gegebenenfalls Angaben über die Quellen und deren Zugänglichkeit enthalten. Die Art und Weise der Information sollte darüber hinaus vorausschauend sein. Und schliesslich muss der Informant jederzeit seine eigenen Interessen offenlegen. Nur wer diese Bedingungen einhält, vermag sein Gegenüber auf nichtmanipulierende Weise in den nötigen Wissensstand zu versetzen, um eine selbständige Entscheidung zu treffen, und respektiert damit dessen Autonomie.

\section{«Ich war noch nie krank.»}

Ein beliebter Einwand gegen die Grippeimpfung lautet: «Ich brauche mich nicht gegen Grippe zu impfen, weil ich nie krank bin, viel Vitamin C zu mir nehme und über ein vorzügliches Immunsystem verfüge. Aus diesem Grunde geht von mir auch keine Ansteckungsgefahr aus.» Bestimmt wäre es nicht vernünftig, sich gegen eine Krankheit impfen zu lassen, die man gar nicht bekommen kann. Dieses Argument sitzt jedoch einer sprachlichen Ungenauigkeit auf. In der deutschen Sprache beispielsweise heissen sowohl die einfache Erkältung als auch die durch Influenzaviren hervorgerufene Atemwegsinfektion Grippe. Das deutsche Wort stammt vom französischen $a b$, und dieses wiederum vom russischen «chripu», das seinerseits Heiserkeit bedeutet [4]. Heiserkeit als gemeinsames Symptom der beiden Krankheiten steht am Anfang einer sprachlichen Undifferenziertheit, durch welche die Influenza mit ihren ernsten Komplikationen verharmlosend mit einer Erkältung gleichgesetzt und in der Konsequenz massiv unterschätzt wird. Vitamin C mag zwar dem Immunsystem im ganzen zugute kommen, schützt aber nicht vor den Influenzaviren. Und selbst der Umstand, dass die Grippe bei etwa 20 bis 30 Prozent der mit Influenzaviren Infizierten nicht ausbricht, kann nicht gegen die Impfung angeführt werden, denn auch von jenen asymptomatischen, klinisch «gesunden» Infizierten wird das Virus weitergegeben. Von diesem Gesichtspunkt her ergibt sich eindeutig der Grundsatz, sich gegen Grippe zu impfen, um die anderen zu schützen.

\section{«Die Impfung macht krank»}

Ein weiteres Argument führt gesundheitliche Bedenken gegen die Grippeimpfung an und verweist auf Geimpfte, die im Anschluss an die Impfung krank geworden sind. Die leichteren Symptome, die sich unmittelbar nach einer Grippeimpfung bei etwa 25 Prozent der Geimpften über ein bis zwei Tage bilden können, stehen allerdings in keinem Verhältnis zu einer Grippeerkrankung. Gravierende Nebenwirkungen, wie beispielsweise bei einer Allergie gegen Hühnereiweiss, stellen sich extrem selten ein. Die Verträglichkeit des Grippeimpfstoffes, der im übrigen ein «Totimpfstoff» ist, konnte in den zurückliegenden Jahren markant verbessert werden. An dieser Stelle mag jene Forderung, sich selber zu informieren, besonders einsehbar werden. Wer indessen realistischen Anlass hat anzunehmen, dass ihm der Impfstoff schaden
Wörterbuch. 4. Band. Leipzig; 1935. Sp. 382-3. 
wird, hat auch einen zu respektierenden Grund, sich nicht impfen zu lassen. Niemand muss sich selber schaden. Allerdings dürfte die Zahl derjenigen, bei denen entsprechende Bedenken begründet sind, sehr klein sein.

\section{«Die Grippeimpfung nützt nichts.»}

Der pragmatische Typus führt gegen die Grippeimpfung das Argument ins Feld, dass sie wirkungslos sei, da man trotz Impfung an Grippe erkranken könne. Jedes Jahr wird die Zusammensetzung des Grippeimpfstoffs von einer Gruppe von Experten der Weltgesundheitsorganisation (WHO) nach einer eingehenden Situationsanalyse neu bestimmt und dabei eine Voraussage über die jeweils im kommenden Winter zu erwartenden Stämme von Grippeviren gemacht. Grundlage dieser Voraussage sind die Ergebnisse aus der weltweiten Grippesurveillance, welche die zirkulierenden Virusstämme im Auge behält. Dank der Optimierung des Systems der Grippesurveillance konnte bei der jährlichen Impfstoffzusammensetzung eine bemerkenswerte Erfolgsquote erreicht werden. Obwohl der Schutz, nach einer Impfung nicht an Grippe zu erkranken, für jüngere Menschen zu 70 bis 90 und für ältere lediglich zu 30 bis 80 Prozent besteht, erweist sich die Impfung auch für diejenigen als sinnvoll, die später trotz Impfung an Grippe erkranken. Bei dieser Gruppe ist nicht nur ein abgeschwächter Verlauf der Krankheit zu beobachten, sondern - was in unserem Zusammenhang nicht ohne Belang ist sie scheiden auch markant weniger Virusmaterial aus, so dass sich die entsprechende Ansteckungsgefahr deutlich vermindert. Von daher vermag das pessimistische, vermeintlich pragmatische Argument keineswegs zu überzeugen.

\section{«Dem Arbeitgeber geht es ja nur um meine Arbeitskraft.»}

Insbesondere ein Teil des Pflegepersonals hegt den Verdacht, dass ihm die Grippeimpfung nur deshalb nahegelegt wird, um die Arbeitsausfälle zu minimieren. Manche Pflegenden glauben sich gegen eine solche Instrumentalisierung mit Impfverweigerung wehren zu müssen. Das entsprechende Verhalten und seine Begründung nähren sich von einem spezifischen Misstrauen gegen die Institution bzw. das «System» und gipfeln in der polemisch überspitzten, etwas bizarr anmutenden Rede vom eigenen «Recht auf Krankheit» [3]. Dem Argument liegt die Erfahrung zugrunde, dass die Pflegenden häufig an
Entscheidungsprozessen zuwenig beteiligt werden und ihnen dabei das Gefühl vermittelt wird, ihre Autonomie würde nicht respektiert. Die Pflegenden vollbringen ihre Arbeit in einem delikaten Spannungsfeld, das sich daraus ergibt, dass oft andere die Entscheidungen treffen, welche die Pflegenden ihrerseits ausführen sollen. Grundsätzlich sollte jedoch niemand etwas tun, mit dem er nicht einverstanden sein oder an dem er sich nicht zumindest kompromissartig beteiligen kann. Die Pflegendenautonomie wird als Problem noch immer zuwenig erkannt und als Herausforderung der Organisationsentwicklung noch immer zuwenig ernst genommen.

\section{Impfkampagnen}

Gutgemeinte Aktionen, den Impfwilligen in Form von Papiertaschentüchern oder eines spendierten Mittagessens eine Aufmerksamkeit zukommen zu lassen, führen mitunter zu Missverständnissen. Das Personal im Medizinalbereich möchte nicht überredet, es möchte überzeugt werden: Echte Beteiligung wendet sich an das Gegenüber als einen autonomen Menschen und informiert sachlich über das in diesem Falle gemeinsame Problem. Wenn sich das Personal nicht impfen lässt, so kann die Institution als ganze nicht alles zur Gefahrenminimierung für die Risikopatientinnen und -patienten unternehmen. Autonomie heisst, sich einem solchen Problem zu stellen und es nach Massgabe der eigenen Moralvorstellungen zu lösen oder sich an dessen Lösung entsprechend zu beteiligen. Was immer in den folgenden Grippesaisons tatsächlich auf uns zukommen mag, müssen die Verantwortungsträger zum Beispiel auch Szenarien bedenken wie die Bewältigung einer Pandemie und die gleichzeitige Betreuung einer gewissen Anzahl von SARS-Kranken. In einem solchen Szenario zeichnen sich bei einem grösseren Ausfall des Personals katastrophale Folgen ab. Für solche Perspektiven müsste es gelingen, gemeinsam getragene Lösungen zu generieren, zu denen die jährliche Grippeimpfung des Personals aus Freiheit und Einsicht gehört. Beteiligung und Engagement für eine Sache können allerdings nicht erst dann eingefordert werden, wenn ich Unterstützung brauche, sondern stellen sich ein, wo Autonomie immer schon respektiert worden ist. Darüber hinaus ist in diesem Zusammenhang schliesslich auch zu überlegen, ob Grippeimpfkampagnen nicht prinzipiell von anderen Instanzen initiiert und durchgeführt werden sollten als vom Arbeitgeber. 


\section{Zusammenfassung}

Gegebenenfalls kann ich zur persönlichen moralischen Einschätzung gelangen, dass es für mich - zum Beispiel aufgrund einer spezifischen Allergie - nicht sinnvoll ist und daher nicht geboten sein kann, mich gegen Grippe zu impfen. Auch weitere moralisch relevante Gründe gegen die Grippeimpfung wären unter spezifischen Umständen vorstellbar. Eine betreffende moralische Entscheidung ist denn jederzeit fraglos zu respektieren. In diesem Sinne kann auch nicht pauschal von einer moralischen Verpflichtung gesprochen werden, sich gegen Grippe impfen zu lassen. Demgegenüber gibt es aber für jeman- den, der seine Autonomie verwirklichen möchte, eine eigentliche Pflicht, jener Frage der Moral nach dem Schutz der anderen mit einer eigenen moralischen Antwort zu begegnen. Mit anderen Worten: Es gibt keine moralische Impfpflicht, aber eine moralische Pflicht, sich mit dem betreffenden Problem auseinanderzusetzen und an der Problemlösung mitzuwirken. In bezug auf die Grippeimpfung - und davon bin ich überzeugt - dürfte der Gebrauch der moralischen Freiheit, die Verwirklichung von Autonomie in der Konsequenz dazu führen, dass sich die Mitglieder des Personals in der überwiegenden Mehrheit zur Grippeimpfung entschliessen.

\section{FMH SERVICES an der}

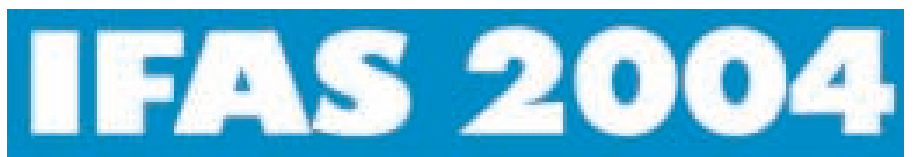

26.-29. Oktober 2004, Messe Zürich

Das detaillierte Programm der FMH-Workshops und des Minikongresses der SGAM sowie ein OnlineAnmeldeformular finden Sie auf der FMH-Website www.fmh.ch. 